\title{
Antonius Weixler
}

\section{Bockwurst, Bier und Zum Zusammenhang von Textschwärzungen und Authentizität}

F. C. Delius' Unsere Siemens-Welt hat vor allem deshalb Literaturgeschichte geschrieben, weil es Prozessgeschichte geschrieben hat. Als die Festschrift zum 125jährigen Bestehen des Hauses S., wie der Text im Untertitel heißt, 1972 erschien, hat der Siemens-Konzern sofort auf Unterlassung, Verkaufsstopp und Schadensersatz geklagt, und was dann nach jahrelangem Prozess über zwei Instanzen und einem Vergleich schließlich ab 1976 mit richterlicher Genehmigung publiziert werden durfte, war eine an insgesamt acht Stellen geschwärzte Version. ${ }^{1}$ In solchen Textschwärzungen zeigt sich hier wie generell oftmals ein Eingriff einer staatlichen Instanz. Geht die Schwärzung auf Anordnung der Judikative zurück, markiert dies in der Regel eine Grenzziehung hinsichtlich der in Art. 5 des Grundgesetzes festgeschriebenen Meinungs- und Kunstfreiheit. Die Schwärzung signalisiert dann, dass die Kunstfreiheit zugunsten von (zumeist) Persönlichkeitsrechten beschnitten werden dürfe und müsse - zumindest in der richterlichen Interpretation. Auch im Prozess um Unsere Siemens-Welt haben die Anwälte der Klageseite damit argumentiert, dass auch ein Großkonzern über Persönlichkeitsrechte verfüge, sodass entsprechend die Kunstfreiheit in diesem Fall eingeschränkt werden müsse. ${ }^{2}$ Diese Strategie war letztlich juristisch erfolgreich wenn auch eher in mäßigem Ausmaß, wenn man die Schwärzung quantitativ bemisst - und doch zugleich ein publicity-Desaster für den Siemens-Konzern.

Noch öfter gehen Schwärzungen in Menge und Fallzahl auf den Eingriff der Exekutive zurück, denn ein Staat lässt sich bekanntlich nur ungern in die Karten schauen, und wenn er schon zur Veröffentlichung von Regierungsdokumenten verpflichtet wird, dann wird versucht, Staatsgeheimnisse vorher unkenntlich zu

1 Delius, F. C. Unsere-Siemens-Welt. Eine Festschrift zum 125jährigen Bestehen des Hauses S. Erweiterte Neuausgabe mit einem Anhang über den Prozeß, über die Kunst der Satire, die Menschenwürde des Konzerns, Bierpreise und den verlorenen Kredit des Hauses S. Mit einem Nachwort von Friedrich Christian Delius. Hamburg: Rotbuch 1995.

2 Eine weitere, nicht erfolgreiche Strategie der Klageseite war sogar der Versuch zu argumentieren, dass auch ein Konzern über die in Art. 1 des Grundgesetztes geschützte Menschenwürde verfüge. Beide Instanzen im Verlauf des Verfahrens, Landgericht wie Oberlandesgericht Stuttgart, sind dieser Argumentation nicht gefolgt. Vgl. Delius: Unsere Siemens-Welt, 216. 
Andere Sparmaßnahmen seien hier als Beispiel dafür genannt, daß auch eine Weltfirma, die Milliarden-Umsätze macht, mit jedem Pfennig rechnen muß, auch wenn er von den eigenen Mitarbeitern kommt. Wenn wir beispielsweise

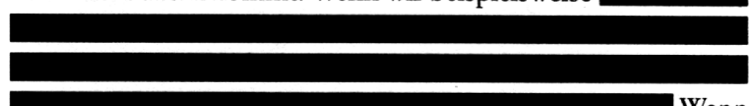

Wenn

wir Broschüren wie »Begegnung mit Siemens", die wir an Besucher des Münchner Siemens-Museums kostenlos verteilen, unseren Mitarbeitern für 5,50 DM anbieten.

Letztendlich gehört auch die Kurspflege unserer Aktien zum Finanzgeschäft. Nachdem wir im April 1972 die Bankexperten und Mitglieder der Vereinigung für Finanzanalysen und Anlageberatung zu einem Informationsgespräch geladen und ihnen eine Gewinnsteigerung für 1972 und 1973 um je 10\% versprochen hatten, zogen unsere Kurse sprunghaft an. Auch das Angebot unserer Optionsanleihe - für jeden Kenner ein todsicheres Geschäft - hat die Siemens-Euphorie an den Börsen und Bankschaltern Europas spürbar beeinflußt.

Abb. 1: Schwärzung in Unsere Siemens-Welt, 100.

machen. Ein verbreitetes Verfahren, das eigentlich auch nur dann besondere Aufmerksamkeit gewinnt, wenn etwas schiefgeht - wie das bei Pentagon-Dokumenten immer wieder der Fall ist ${ }^{3}$ oder 2017 auch beim Abschlussbericht des deutschen Geheimdienst-Untersuchungsausschusses; ${ }^{4}$ oder wenn die Streichungen so exzessiv ausfallen, dass von ,Veröffentlichen' kaum (oder nur noch in Anführungszeichen) gesprochen werden kann. In jüngster Zeit haben für Letzteres vor allem der Muller-Report, ${ }^{5}$ der im April 2019 Donald Trumps vermeintliche, von Russland unterstützte Wahlkampfmanipulation offenlegen sollte, sowie Mohamedou Ould Slahis Guantanamo-Tagebuch (2015) ${ }^{6}$ für Aufsehen gesorgt.

3 Vgl. o. V. „Geschwärztes PDF-Dokument blamiert Pentagon“. In: DerStandard.de vom 02.06.2005. https://www.derstandard.de/story/2034120/geschwaerztes-pdf-dokument-

blamiert-pentagon (01.03.2021).

4 Vgl. Meister, Andre. „Wir veröffentlichen den Abschlussbericht - ohne die Schwärzungen“. Netzpolitik.org vom 24.06.2017. https://netzpolitik.org/2017/geheimdienst-untersuchungs ausschuss-wir-veroeffentlichen-den-abschlussbericht-ohne-die-schwaerzungen/ (01.03.2021). 5 In dem insgesamt 448 Seiten umfassenden Muller-Report waren insgesamt 7,25 Prozent der Textstellen geschwärzt.

6 Slahi, Mohamedou Ould. Das Guantanamo-Tagebuch [Guantánamo Diary]. Stuttgart: Cotta 2015. 


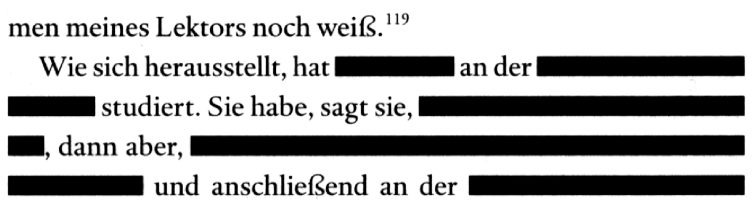

Seminare in belegt, Auslandssemester in verbracht, bevor sie ins Verlagswesen eingestiegen ist. Jetzt arbeitet sie als Übersetzerin und schreibt an einer Doktorarbeit über das Motiv der Unschuld im Werk von

Wir gehen wieder nach draußen, stellen uns an die Balustra-

Abb. 2: Schwärzung in Tod in Turin, 190.

40 Jahre nach dem Siemens-Delius-Prozess greift Jan Brandt das Verfahren der Schwärzung in seiner Autofiktion Tod in Turin 2015 wieder auf, und auch hier erfolgen die Schwärzungen nicht aus irgendeinem, sondern erneut aus ,persönlichkeitsrechtlichen Gründen“7. Und doch könnte der Unterschied zwischen diesen beiden literarischen Schwärzungsfällen größer kaum sein. Zwar handelt es sich in beiden Fällen um Hybridtexte an der Schwelle von Fakt und Fiktion, doch basiert in Delius' "Dokumentarsatire“8 ${ }^{8}$ das erkennbar in einem verfremdenden Stil Beschriebene dennoch auf Dokumenten - zumindest wurde gerade diese Faktualität des Dokumentarischen auch in der Dokumentarsatire zum zentralen Streitpunkt in der Gerichtsverhandlung -, während in Brandts Autofiktion die beschriebenen Erlebnisse vom autobiographischen Erzähler-Ich ausreichend beglaubigt (oder ausreichend fiktionalisiert) hätten werden können. Bei Delius wird die Schwärzung richterlich oktroyiert und ist fortan conditio sine qua non jeder Publikationsmöglichkeit, bei Brandt sind die „persönlichkeitsrechtlichen Gründe“ nur fingiert. Zudem wird die Schwärzung vor der Erstpublikation vorgenommen, ein gerichtlicher Eingriff wird damit lediglich als fiktive Möglichkeit antizipatorisch vorgeführt. Letztlich bleibt auch unklar, welche Instanz die Schwärzung bei Brandt zu verantworten hat - ein Aspekt, der gleich noch genauer zu betrachten sein wird.

7 Brandt, Jan. Tod in Turin. Eine italienische Reise ohne Wiederkehr. Köln: DuMont 2015, 5 [an dieser Stelle ohne Paginierung].

8 Delius: Unsere Siemens-Welt, 187 et passim. 

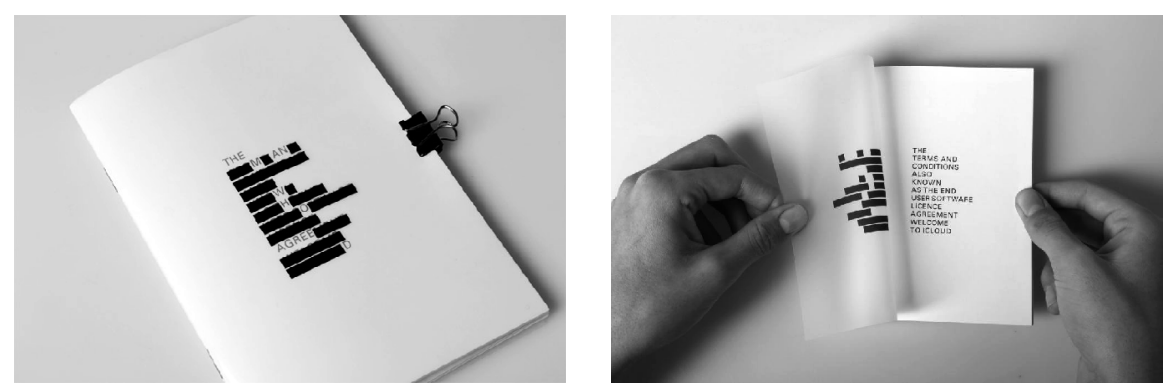

Abb. 3 und 4: Florence Meuniers The Man Who Agreed.

Eine gänzlich andere Spielart der Textschwärzung führt Florence Meunier 2015 mit The Man Who Agreed vor. ${ }^{9}$ Meunier nimmt sich einen dieser juristischen Texte vor, die wir alle mittlerweile in unserem digitalen Zeitalter beinahe täglich vorgelegt bekommen und wohl nahezu immer ungelesen unterschreiben und akzeptieren, hier konkret die Allgemeinen Geschäftsbedingungen des Cloud-Speicherdienstes von Apple. Meunier streicht diese Geschäftsbedingungen so zusammen, dass aus dem faktual-juristischen Kauderwelsch eine (letztlich vielleicht gar nicht so) fiktionale Kurzgeschichte entsteht.

Alle drei hier vorgestellten Beispiele lassen sich in einem weiteren Sinne als Dokufiktionen bezeichnen, da stets Dokumente mit literarischen Verfahren bearbeitet, vorgeführt oder erzeugt werden. Grundlage oder Basis einer Dokufiktion, gleichsam ihr Rohstoff oder Ausgangsmaterial, ist damit stets das Dokument (als Text oder Material) oder das Dokumentarische (als Verfahren), mit dem dann in mal mehr, mal weniger fiktionalisierender Weise umgegangen oder das um fiktionalisierende Verfahren ergänzt wird. In Dokufiktionen geht es „gerade um die Gleichzeitigkeit von referentiellem und konstruktivem, von dokumentarischem und nicht-dokumentarischem Zugang zum verwendeten Ausgangsmaterial und -stoff. " ${ }^{10}$ In der Forschung zur Dokufiktion wird denn auch zumeist die spezifische Hybridität der Texte zwischen Dokument(ation) und Fiktion untersucht, der Textstatus der zugrundeliegenden Dokumente wird dabei allerdings oft als „[e]chtes

9 Meunier, Florence. The Man Who Agreed. Ohne Verlagsangabe 2015. Hier und im Folgenden zitiert nach: https://blog.supertext.ch/2015/01/lesenswert-wie-aus-den-16-seitigen-icloud-agbeine-kurzgeschichte-wurde/ (01.03.2021).

10 Bidmon, Agnes. „Geschichte(n) zwischen Fakt und Fiktion. Formen und Funktionen dokufiktionalen Erzählens in Jugendbüchern von Dirk Reinhardt: Edelweißpiraten und Train Kids.“ In: Fakt, Fake und Fiktion. Jahrbuch der Gesellschaft für Kinder- und Jugendliteraturforschung 2019. Hg. Gabriele von Glasenapp u. a. http://www.gkjf.de/jahrbuch-2019-open-access/, 63-77, hier 64. 
dokumentarisches Material“"11 in seiner historischen Zeugenschaft ${ }^{12}$ fraglos akzeptiert. Ziel dieses Beitrages soll sein, einige Überlegungen zu diesem Textstatus des Dokumentes anzustellen. ${ }^{13}$ Ein Textstatus, so soll gezeigt werden, der sich am Beispiel der Textschwärzungen ganz besonders prägnant untersuchen lässt. Denn in den eingangs vorgestellten Beispielen zeigt sich an ein und demselben Verfahren - der Schwärzung von gedrucktem Textmaterial - ein sehr unterschiedlicher Umgang mit diesem Textstatus (oder auch dem Medium) Dokument sowie mit dem Verfahren der Dokumentation. Ja, noch entscheidender scheint zu sein, dass der Status des Textes als Dokument hier je ein anderer ist, und dieser Status hängt wiederum wesentlich mit dem Verfahren der Schwärzung zusammen. Um dies zu untersuchen, sind zunächst einige Überlegungen zum Zusammenhang von Autorisierung, Authentifizierung und dem Dokumentenstatus anzustellen, bevor die drei eingangs skizzierten Beispiele noch einmal näher untersucht werden.

\section{Das Dokumentarische am Dokument: zum Verhältnis von Authentizität und Dokument}

Sowohl der Textstatus als auch das Verfahren der Dokumentation sind für die Gattung Dokufiktion deshalb so zentral, weil an ihnen als Bezugspunkt oder Beglaubigungsgegenstand die Wahrhaftigkeit, die Originalität oder - was zumeist dasselbe meint - die Authentizität der fiktiven und/oder fiktionalen Anteile der dokufiktionalen Erzählungen verbürgt oder autorisiert werden müssen. Dabei wird der Status eines Dokumentes als Dokument meist nicht hinterfragt, sondern oftmals als selbstverständlich vorausgesetzt und auch in der Rezeption tendenziell kritiklos akzeptiert. Das liegt daran, dass Dokumente Schriftstücke oder Objekte mit außergewöhnlicher Wirkmächtigkeit sind. Da sie in Dokufiktionen zumeist die

11 Wiegandt, Markus. Chronisten der Zwischenwelten. Dokufiktion als Genre. Operationalisierung eines medienwissenschaftlichen Begriffs für die Literaturwissenschaft. Heidelberg: Winter 2017, 45. 12 Assmann, Aleida. „Vier Grundtypen von Zeugenschaft“. In: Zeugenschaft des Holocaust. Zwischen Trauma, Tradierung und Ermittlung. Hg. Fritz Bauer Institut. Frankfurt a. M.: Campus 2007, 33-51, hier 39. Vgl. dazu auch Bidmon: „Geschichte(n) zwischen Fakt und Fiktion“, 68.

13 Bei Wiegandt findet sich ein Kapitel, das sich mit der „Bestimmung des Dokumentbegriffs“ beschäftigt und diesen vor allem etymologisch erläutert. Er konstatiert aber auch, dass Dokumente „keineswegs die Tatsachen selbst [bedeuten], vielmehr scheinen sie als Darstellungen im literarischen Kontext auf die faktuale Wirklichkeit zu verweisen. "Wiegandt: Chronisten der Zwischenwelten, 18. 
Funktion haben, die fiktionalen Erzählelemente zu beglaubigen, soll mittels der Präsentation von Dokumenten oder dem ausgestellten Verfahren der Dokumentation die gesamte Erzählung trotz ihrer Hybridität zwischen Fakt und Fiktion in der Rezeption authentisch wirken. Das Verhältnis von Dokument, Autorität und Authentizität ist aber mitnichten so einfach und schon gar nicht so kausal und/oder chronologisch zu verstehen wie hier gerade beschrieben. Ihr Verhältnis ist vielmehr ausgesprochen kompliziert und gleicht eher der Geschichte von der Henne und dem Ei. Das ist schon in der Begriffsgeschichte so: ,Authentizität‘ entsteht als Konzept historisch durch eine Institution oder Person, die über die Autorität verfügt, ein Dokument authentifizieren zu können. ${ }^{14}$ Klassisch ist dies etwa der Fall, wenn die Kirche die eine richtige Bibelauslegung als kanonisch autorisiert und damit authentifiziert. Sichtbarer Ausweis dieses Prozesses ist die fast schon sprichwörtliche Kombination aus Amt und Siegel (oder Stempel). Auch die Autorschaft ist etymologisch eng an Autorität und Authentizität gebunden: lat. auctor bezeichnet die*denjenigen, die/der auctoritas besitzt, d. h. eine Person oder Institution, die etwas autorisieren und authentisieren kann; ${ }^{15}$ historisch waren das eben diejenigen Personen, die über ein Siegel verfügten. Im amtlichen Siegel lebt dieser Autoritätsaspekt bis heute fort: Ein Siegel autorisiert eine Person, aus einem normalen Blatt Papier ein offizielles Dokument machen zu können. So wie ein Dokument also der autorisierenden und authentifizierenden Bestätigung bedarf, wird umgekehrt - so könnte man diese historische Kernkonstellation interpretieren - eine gewöhnliche Person (oder Institution) erst dadurch zu einer Autorität, wenn sie über die Macht und Ausstattung verfügt, etwas dokumentieren und authentifizieren zu können. Wenn wir diesen Prozess aber als einen derartig sich selbst erzeugenden, also als einen im besten Sinne autopoietischen Effekt verstehen, hat dies wiederum Folgen für die sowohl theoretische als auch analytische Betrachtung von Dokumenten und von der auf diesen Dokumenten basierenden Erzählform der Dokufiktion.

Damit lassen sich in der Systematik der Begriffe bzw. Phänomene ,Dokument‘ und ,Authentizität' einige Strukturanalogien erkennen. An anderer Stelle wurde bereits versucht ausführlicher darzulegen, dass es Authentizität in einem essen-

14 Vgl. Röttgers, Kurt/Fabian, Reinhard. „Authentisch“. In: Historisches Wörterbuch der Philosophie. Hg. Joachim Ritter. Darmstadt: Wissenschaftliche Buchgesellschaft 1971, Bd. I, Sp. 691-692, hier 691.

15 Aus dem Lateinischen auctoritas wird beispielsweise das Adjektiv authenticus. Vgl. Röttgers/ Fabian: „Authentisch“, 691, und Martínez, Matías. „Zur Einführung. Authentizität und Medialität in künstlerischen Darstellungen des Holocaust“. In: Der Holocaust und die Künste. Medialität und Authentizität von Holocaust-Darstellungen in Literatur, Film, Video, Malerei, Denkmälern, Comic und Musik. Hg. Matías Martínez. Bielefeld: Aisthesis 2004, 7-21, hier 12. 
zialistischen Sinne nicht gibt, dass lediglich durch bestimmte Erzählverfahren angeregt werden kann, einem Text, einem Bild oder einem anderen medialen Zeichenträger in der Rezeption die Qualität der Authentizität zuzuschreiben. ${ }^{16} \mathrm{Im}$ Folgenden soll in diesem Beitrag gezeigt werden, dass Gleiches auch für Dokumente gilt, dass es also auch Dokumente als authentische Zeugnisse von realen Ereignissen in einem essenzialistischen Sinne nicht gibt, sondern dass sie gleichsam erst als solche produziert - oder konstruktivistisch formuliert - konstruiert werden müssen. Die eingangs genannten und in der zweiten Hälfte dieses Beitrages noch einmal näher zu betrachtenden Schwärzungsverfahren dienen dabei als Beispiele für drei unterschiedliche Verfahren einer solchen Konstruktion.

Bevor dies näher betrachtet wird, sei noch eine etwas grundsätzlichere Bemerkung zur erzähltheoretischen Einordnung der Dokufiktion als Hybridgattung zwischen fiktionalem und faktualem Erzählen vorangestellt: Generell spielt die Authentifizierung der Darstellung selbstredend in allen Formen des faktualen Erzählens eine zentrale Rolle. Grundsätzlich gilt: Wenn wir faktualem Erzählen glauben, d. h. wenn wir den genannten Fakten in der Rezeption Authentizität zuschreiben, bedeutet das nichts anderes, als dass die Beglaubigung/Autorisierung der genannten Daten (Ereignisse) funktioniert hat und diese von den Rezipient*innen als reale Fakten rezipiert werden. ${ }^{17}$ Das ist der ,referenzielle Pakt ${ }^{\star}$ (oder faktuale Pakt). Unterm Strich heißt das ja nichts anderes, als dass bei aller Wahrscheinlichkeit der Referenz die abschließende Zuschreibung eines Textes zu einer faktualen Gattung letztlich nur aus einer Behauptung des Textes gefolgert werden kann. Einer Behauptung kann man aber auch misstrauen. Der ,referenzielle Pakt ' beruht also nicht nur auf einer Referenzbehauptung im Text, sondern auch

16 Weixler, Antonius. „Authentisches erzählen - authentisches Erzählen. Über Authentizität als Zuschreibungsphänomen und Pakt.“ In: Authentisches Erzählen. Produktion, Narration, Rezeption. Hg. Antonius Weixler. Berlin/Boston: De Gruyter 2012, 1-32.

17 Vgl. Martínez: „Zur Einführung“, 7-21; vgl in diesem Zusammenhang auch Knaller, Susanne. „Genealogie des ästhetischen Authentizitätsbegriffs“. In: Authentizität. Diskussion eines ästhetischen Begriffs. Hg. Susanne Knaller, Harro Müller. München/Paderborn: Fink 2006, 17-35; Knaller, Susanne. Ein Wort aus der Fremde. Geschichte und Theorie des Begriffs Authentizität, Heidelberg: Winter 2007. Für die Autobiografie hat das auch schon Philippe Lejeune erkannt, dessen „autobiographischer Pakt“ illokutionstechnisch v. a. ein „referentieller Pakt“ ist, in dem die`der Autor`in die Referenz autorisiert und die Rezipient`innen diesem Referenzverhältnis glauben. Frank Zipfel weist entsprechend darauf hin, dass der autobiographische Pakt damit nur eine andere Formulierung für die grundlegende Regel faktualen Erzählens bzw. „die allgemeinen Referenzregeln der Sprache“ ist, d. h. den Behauptungsregeln Glauben zu schenken, wie sie John R. Searle oder den Kommunikationsregeln wie sie Paul Grice „ausformuliert haben“. Zipfel, Frank. „Autofiktion. Zwischen den Grenzen von Faktualität, Fiktionalität und Literarität?“ In: Grenzen der Literatur. Zum Begriff und Phänomen des Literarischen. Hg. Simone Winko, Fotis Jannidis, Gerhard Lauer. Berlin/New York: De Gruyter 2009, 287-314, hier 288. 
auf der Bereitschaft der Leser`innen, dieser diskursiven, rhetorischen Behauptung Glauben zu schenken. In diesen vorangehenden Beschreibungen wurde bewusst neutral formuliert im Hinblick auf die Instanz, die diese Autorisierung vornimmt. In den allermeisten Fällen ist für die Autorisierung die Autorinstanz zuständig. Mit anderen Worten: Wenn wir faktualem Erzählen Glauben schenken und die Fakten als authentisch rezipieren, dann bedeutet das in der Regel nichts anderes, als dass die Autorisierung der genannten Daten durch die Autorinstanz funktioniert hat. ${ }^{18}$ Doch die Autorinstanz ist nicht der einzige Bezugspunkt einer Autorisierung und Authentifizierung. Gerade im dokumentarischen wie im dokufiktionalen Erzählen wird der referenzielle Pakt in bedeutsamem Ausmaß durch die Authentizität und Autorität erzeugt, die wir historischen Objekten oder Dokumenten zuschreiben - ein Phänomen, das entsprechend einmal als „Objektauthentizität“ und einmal als „Referenzauthentizität“ benannt wurde. ${ }^{19}$

Um im Folgenden den Versuch zu unternehmen, diese etwas ,naive“ (durchaus im Schiller'schen Sinne) Vorstellung von authentischen Dokumenten näher $\mathrm{zu}$ betrachten, wollen wir zu Beginn (und probehalber) eine radikalkonstruktivistische Position einnehmen: Als Ausgangspunkt soll die Vorstellung dienen, dass kein Dokument per se ein authentisches Dokument sein kann. Jedes Dokument bedarf der Autorisierung durch eine zur Autorisierung befähigte Instanz, erst durch vorausgehende Verfahren der Autorisierung bzw. Authentifizierung erhalten Dokumente ihren Status, erst dadurch unterscheiden sie sich von einem gewöhnlichen Blatt Papier. Selbst ein archäologisches Fundstück, das, so mag ein Einwand lauten, durchaus über eine ontologische oder ontische Qualität des Authentischen verfügt, hat diese Qualität nicht an und für sich. Auch solchen Fundstücken können wir diese Qualität in der Rezeption erst dann zuschreiben, wenn ihr Status als Dokument (bzw. archäologisches Fundstück aus einer bestimmten Epoche) durch empirische Methoden nachgewiesen und durch entsprechende Instanzen, die über solche Messmethoden verfügen und die Autorität besitzen, diese Methoden auch korrekt anzuwenden, bestätigt wurde. Am Ende bedarf es also immer eines Sigels, das aus einem gewöhnlichen Fundstück oder einem herkömmlichen Blatt Papier ein Dokument mit Referenzauthentizität macht.

Gleiches gilt für das Hochwertphänomen der Authentizität generell, sodass sich, wie erwähnt, einige Strukturanalogien beobachten lassen. Authentizität gibt es nicht, es ist weder eine ontologische noch eine ontische Qualität; es gibt nur narrative bzw. diskursive Verfahren, die anregen können, dass wir in der

18 Vgl. Martínez: „Zur Einführung“.

19 Vgl. Weixler: „Authentisches erzählen - authentisches Erzählen“, 17-22; Knaller: Ein Wort aus der Fremde, 8-22. 
Rezeption einem Text, einer Person etc. Authentizität zuschreiben, also einen Authentizitätspakt schließen. Für dieses Rezeptionsphänomen wurde, wie bereits erwähnt, an anderer Stelle und etwas ausführlicher eine Differenzierung von Authentizitätsbegriffen vorgeschlagen; diese Differenzierung lässt sich wiederum (nicht gänzlich, aber doch) einigermaßen strukturanalog (und ist dann ahistorisch und vor allem systematisch zu verstehen) auf den Dokumentenstatus übertragen. Differenzieren lassen sich demnach erstens eine tendenziell vormoderne oder alltagssprachliche Vorstellung einer referenziellen Authentizität der Zuweisung, zweitens eine tendenziell moderne Vorstellung einer relationalen Authentizität der ,Erschreibung' und drittens postmoderne Verfahren einer relationalen Authentizität der Zuschreibung. Formelhaft lässt sich dies wie folgt darstellen:

$\begin{array}{ll}\text { referenzielle Authentizität der Zuweisung } & \mathrm{T}=\mathrm{W} \\ \text { relationale Authentizität der ,Erschreibung } & \mathrm{T} \rightarrow \mathrm{W} \\ \text { relationale Authentizität der Zuschreibung } & \mathrm{T} \neq \mathrm{W}\end{array}$

Die erste Form entspricht der auch heute noch gültigen umgangssprachlichen Bedeutung, bei der eine direkte Verbindung zwischen Bezeichnetem und Bezeichnendem angenommen wird. In der Formel steht das T somit für ,Text‘, das W kann wiederum für ,Welt‘, ,Wirklichkeit“ oder ,Wahrheit‘ stehen, das =-Zeichen steht für Referenz. Anders formuliert: Bei einer referenziellen Zuweisung (oder „Referenzauthentizität“) wird einem sprachlichen Zeichen die ontologische Qualität eines Objektes zugewiesen. Bei der zweiten Form wird zwar bereits erkannt, dass es sich bei der Verbindung zwischen Signifikat und Signifikant immer um eine mediale Relation handelt, eine direkte Referenzverbindung also nicht möglich ist, aber immer noch angenommen wird, dass diese Relation relativ unproblematisch herzustellen, d. h. zu ,erschreiben“ ist (in der Formel durch den Pfeil symbolisiert). In der dritten Variante, welche die ersten beiden Konzepte hinterfragt, wird Authentizität als ein Phänomen verstanden, das lediglich durch Verfahren der Produktion und/oder Narration angeregt werden kann, indem einer medialen Kommunikation in der Rezeption das Prädikat ,authentisch' zugeschrieben wird..$^{20}$ Mit diesen Authentizitätstypen ist keine historische Entwicklung beschrieben; die drei Konzepte lösen einander nicht ab, sondern sind alle auch heute noch in Gebrauch. In der umgangssprachlichen Verwendung des Begriffs findet sich etwa nach wie vor die Vorstellung der referenziellen Zuweisung. Referenzielle Authentizität ist damit

20 Vgl. Weixler: „Authentisches erzählen - authentisches Erzählen“. Findet also bereits in der zweiten Form eine Verschiebung vom Inhalt zur Darstellung statt, verschiebt sich dies in der dritten noch weiter in die Rezeption. 
ein Phänomen, das relativ unverändert durch die Postmoderne hindurchgewandert ist, wohingegen das relationale Zuschreibungsphänomen Authentizität ein die Bedingungen der Postmoderne reflektierendes Phänomen darstellt. ${ }^{21}$ Dahinter steckt die durchaus intrikate Wirkmächtigkeit des Authentischen: So kann uns in der heutigen massenmedialen Kommunikation das Phänomen eigentlich nur in der letzten Variante begegnen, allerdings schreiben wir im Rezeptionsprozess den Gegenständen dann aber die Qualität der referenziellen Authentizität zu.

Um diese Differenzierung nun auf Dokufiktion im Allgemeinen und den Dokumentenstatus im Besonderen anzuwenden, können wir in einem ersten Schritt zunächst das „,“ für ,Text“ durch ein „D“ für ,Dokument“ ersetzen. Natürlich kann man Texte und Dokumente nicht einfach gleichsetzen bzw. das eine durch das andere ersetzen, es gibt ja auch faktuale Texte, die aus Dokumenten zitieren. ${ }^{22}$ Es soll im Folgenden aber vor allem um dokufiktionale Texte gehen, die die Dokumente in ihrer Materialität zeigen. Im Bewusstsein der jeder Formel zwangsläufig inhärenten Gefahren der Simplifizierung lässt sich das obige Schema dann wie folgt auf Dokumente übertragen:

referenzielle Authentizität:

$\mathrm{D}=\mathrm{W}$

relationale Authentizität der Erschreibung:

$\mathrm{D} \rightarrow \mathrm{W}$

relationale Authentizität der Zuschreibung:

$D \neq W$

21 Damit wird hier explizit eine Gegenposition zu Stimmen eingenommen, die in der gegenwärtigen Konjunktur des Authentizitätsbegriffes das Ende der Postmoderne zu erkennen glauben. Als Epochenwende gilt diesen Positionen der Terroranschlag vom 11. September, etwa für Ulla Haselstein, Andrew Gross und MaryAnn Snyder-Körber, die resümieren: „The attacks seemed to be authentic in a way, that postmodernism denied.“ (Haselstein, Ulla/Gross, Andrew S./Snyder-Körber, MaryAnn. „Introduction: Returns of the Real“. In: The Pathos of Authenticity. American Passions of the Real. Hg. Ulla Haselstein, Andrew S. Gross, MaryAnn Snyder-Körber. Heidelberg: Winter 2010, 9-32, hier 15). Auch Wolfgang Funk und Lucia Krämer verstehen Authentizität als einen „Schlüsselbegriff nach-postmodernen Denkens und Fühlens“ (Funk, Wolfgang/Krämer, Lucia. „Fiktionen von Wirklichkeit - Authentizität zwischen Materialität und Konstruktion“. In: Fiktionen von Wirklichkeit - Authentizität zwischen Materialität und Konstruktion. Hg. Wolfgang Funk, Lucia Krämer. Bielefeld: transcript 2011, 7-24, hier 7). Als prominente Gegenstimme ist demgegenüber Jean Baudrillard anzuführen, der 9/11 v. a. als symbolisches Bildereignis versteht: „Wie steht es aber um das reale Ereignis, wenn überall das Bild, die Fiktion, das Virtuelle die Realität prägen? [...] Doch übertrifft die Realität wirklich die Fiktion? Wenn dies der Fall zu sein scheint, dann nur deshalb, weil die Realität die Energie der Fiktion absorbiert hat und selbst zur Fiktion geworden ist.“ Baudrillard, Jean. „Der Geist des Terrorismus. Herausforderung des Systems durch die symbolische Gabe des Todes“. In: Der Geist des Terrorismus. Hg. Peter Engelmann. Wien: Passagen Verlag 2002, 11-35, hier 30.

22 Vgl. zur Differenzierung von Dokument und Text auch Wiegandt: Chronisten der Zwischenwelten, 16-20. 
Mit diesem Schema kann folglich die Wirkmächtigkeit von Dokumenten (nicht nur) im dokufiktionalen Erzählen erklärt werden: Die umgangssprachliche Wirkung von Dokumenten liegt darin, dass sich in ihnen eine unmittelbare empirisch-ontologische Referenzialität auf die Welt/Wirklichkeit ausdrückt, wie dies archäologischen Fundstücken auch zu eigen ist - insofern ist die oben probehalber angeführte, radikalkonstruktivistische Position hiermit schon wieder zurückzunehmen. Wichtig an dieser Übersicht erscheint überdies die Verbindung mit der mittleren Formel, mit der die Einsicht ausgedrückt wird, dass Dokumente selbstredend durchaus Beweiskraft besitzen, dass Dokumente aber erst durch einen Prozess der ,Erschreibung' bzw. Autorisierung oder Authentifizierung zu einem Dokument gemacht werden müssen. In einer erweiterten Formel ließe sich dies auch als $\mathrm{D} \leftarrow \mathrm{A} \rightarrow \mathrm{W}$ darstellen. Wenn archäologischen Fundstücken beispielswiese eine referenzauthentische Qualität zugeschrieben wird, so wird oftmals vergessen, dass es für die Erlangung dieser Qualität einer Autorisierung gemäß dieser mittleren Formel bedarf. In der relationalen Form des dokufiktionalen Erzählens werden eine oder beide Seiten dieser Formel sodann als nicht unproblematisch ausgestellt, d.h. es wird die Unmöglichkeit einer ,reinen' Referenzauthentizität eines Dokumentes und/oder der medialen Kommunikationsform thematisiert.

Wenn medialen Kommunikationsformen heute generell misstraut wird, bedeutet das nichts anderes, als dass wir uns bewusst sind, dass es in medialen Kommunikationsformen keine Referenzauthentizität geben kann. Zuschreibung bedeutet dann, dass durch eine kontextuelle, meist eben narrative ,Konstruktion eine Zuschreibung des Qualitätsmerkmals Authentizität angeregt werden muss. Eine sehr wirksame Möglichkeit, eine solche Zuschreibung herzustellen, besteht darin, gerade die Unmöglichkeit von herkömmlicher Authentizität selbstreflexiv zu thematisieren. Christian Huck umschreibt diesen Aspekt wie folgt: „Authentifizierung [kann] jetzt nur noch durch den selbstreflexiven Bruch mit Authentifizierungsregeln erreicht werden". ${ }^{23}$ Solche selbstreflexiven Formen lassen sich entsprechend auch als meta-authentisches Erzählen bezeichnen. Auf das dokufiktionale Erzählen übertragen kann dies wiederum erklären, warum ein „,semidokumentarischer Vertrag“24 authentischer wirken kann als eine ,reine“ Dokumentation. Um eine solche Metaauthentizität herzustellen, stellen viele Erzählungen eine Abweichung von der Norm des herkömmlichen authentischen Erzählens aus. Mit Huck lassen sich solche Normabweichungen allerdings als „falscher Umkehrschluss" beschreiben:

23 Huck, Christian. „Authentizität im Dokumentarfilm. Das Prinzip des falschen Umkehrschlusses als Erzählstrategie zur Beglaubigung massenmedialen Wissens“. In: Authentisches Erzählen. Produktion, Narration, Rezeption. Hg. Antonius Weixler. Berlin/Boston: De Gruyter 2012, 239-264. 24 Bidmon: „Geschichte(n) zwischen Fakt und Fiktion“, 69 [i. O. mit Herv.]. 
Indem diese Differenz zu den als verfälschend angesehenen Massenmedien hervorgehoben wird, soll dem Zuschauer folgender Umkehrschluss nahegelegt werden: Wenn die Verfahren der Massenmedien angewendet werden, dann wird verfälscht und verstellt. Ergo: Wenn die Verfahren der Massenmedien nicht angewendet werden, dann wird nicht verfälscht und nicht verstellt. ${ }^{25}$

Mit anderen Worten: Wenn gezeigt wird, was sonst nicht gezeigt wird, dann wird scheinbar „alles gezeigt“. Dies wiederum ist ja genau das, was Dokufiktionen von herkömmlichem faktualen Erzählen unterscheidet. Indem fiktionalisierende Erzählverfahren verwendet werden und indem diese als solche auch explizit ausgestellt werden, um damit auf die Unmöglichkeit einer rein referenziellen Authentizität hinzuweisen, werden die Rezipient*innen zugleich für diese Unmöglichkeit besonders sensibilisiert. In vielen dokufiktionalen Erzählformaten werden daher Dokumente einerseits in ihrer Materialität vorgeführt, andererseits dabei selten bloß ,naiv‘ präsentiert. Das in Reenactments Dargestellte, wie sie sich in filmischen Dokufiktionen praktisch immer finden, wird beispielsweise durch die gezeigten Dokumente beglaubigt und autorisiert, zugleich werden aber eben nicht nur die Dokumente alleine gezeigt, sondern es wird auf die emotional stärker wirkenden Reenactments zurückgegriffen. Darüber hinaus wird nicht selten durch erzählerische Verfahren explizit vorgeführt, warum es sich bei den Dokumenten überhaupt um Texte mit einem solchen Status handelt. Die Vorführung des Dokumentenstatus in seiner Materialität zeigt sich an Textschwärzungen aber ganz besonders intrikat, wie nun im zweiten Teil dieses Beitrages gezeigt werden soll.

\section{Der Fall Delius oder das Persönlichkeitsrecht eines Großkonzerns}

Die Ehre eines deutschen Großkonzerns wird an drei Dingen verteidigt: an Bockwurst, Bier und Auschwitz. So könnte man, natürlich etwas verkürzend und polemisch, die Prozessgeschichte um Delius' Unsere Siemens-Welt aus einer Zeit, als die Bücher noch geholfen haben, zusammenfassen. In diesem Fall wollte Siemens durch Klage auf Unterlassung die Veröffentlichung des gesamten Buches verhindern. Zur Strategie des Konzerns und seiner Anwälte gehörte es dabei unter anderem, dass der bis dato noch relativ unbekannte Autor und der relativ kleine Rotbuch Verlag schlichtweg durch die Verfahrenskosten finanziell ruiniert und so zum Einlenken gezwungen werden sollten. Als diese Strategie nicht verfing, lief

25 Huck: „Authentizität im Dokumentarfilm“, 251. 
das Verfahren letztlich darauf hinaus, dass die Behauptungen zur historischen Entwicklung des Siemens-Konzerts vom Gericht auf ihre Richtigkeit hin überprüft wurden, bzw. genauer: Autor und Verlag deren Richtigkeit nachweisen mussten. Geschwärzt werden mussten dann in der Neuauflage von 1976 jene Passagen, die Delius nicht mit Dokumenten belegen konnte.

Doch der Reihe nach. Um zu verstehen, was der Prozess und der diesen abschließende Vergleich mit dem Text gemacht haben, müssen wir uns zunächst vergegenwärtigen, wie der Status der satirischen Festschrift vor Beginn des Gerichtsverfahrens zu bewerten ist. Unsere Siemens-Welt ist als „Dokumentarsatire“ die Parodie einer Festschrift - man könnte auch sagen: ein als Eloge verkleidetes Pasquill - und als solche zweifelsohne ein bedeutendes Beispiel der dokumentarischen Literatur der 1970er Jahre, eine Strömung, die eine wichtige Vorläuferin der zeitgenössischen dokufiktionalen Literatur ist, von der letztere aber üblicherweise auch aufgrund ihres deutlich stärkeren hybriden Status abgegrenzt wird. ${ }^{26}$ Müssen die in einer Satire präsentierten Daten damit aber stets auch belegbare Fakten sein? Delius nimmt im mit „Zum Geleit“ überschriebenen Vorwort zur ersten Auflage für sich in Anspruch, „,keine Zahl, kein Faktum, keinen Vorgang erfun$\operatorname{den}^{\text {“27 }} \mathrm{zu}$ haben. Nach der Klage von Siemens muss er diesen Referenzcharakter des Textes in einem veränderten Vorwort wie folgt relativieren:

Das vorliegende Buch ist weder von der Siemens AG autorisiert noch in ihrer Verantwortung geschrieben. Es handelt sich vielmehr um einen freiwilligen Festbeitrag eines freien Siemens-Forschers zum 125jährigen Bestehen dieses Unternehmens. Natürlich kann dieses Buch die Vorteile einer autorisierten Schrift des Hauses Siemens weder bieten noch ersetzen. [...] Zahlen, Fakten, Vorgänge wurden entsprechend dem satirischen Charakter der Schrift zum Teil erfunden, zum Teil aus Veröffentlichungen wörtlich oder in veränderter Form übernommen. ${ }^{28}$

Nach Beendigung des Gerichtsverfahrens kann Delius 1976 ein zweites Vorwort voranstellen, um die im ersten Geleitwort vorgenommene Einschränkung wieder zu kassieren. Nachdem dort zunächst beschrieben wird, dass der Passus von den zum Teil erfundenen „Zahlen, Fakten, Vorgängen“ nur auf den Druck der Siemens-Anwälte zurückgeht, heißt es sodann:

Damit der Leser nicht den gleichen Mißverständnissen [wie die Verantwortlichen im Hause Siemens] erliegt, erklärt der Verfasser hier noch einmal: Das diesem Buch zugrundeliegende Material war so reichhaltig, daß der Verfasser nicht jede der Tausende von Einzelinforma-

26 Vgl. Wiegandt: Chronisten der Zwischenwelten, 64-70.

27 Delius: Unsere Siemens-Welt, 201.

28 Delius: Unsere Siemens-Welt, 9-10. 
tionen auf ihre Richtigkeit überprüfen konnte, andererseits auch nicht genötigt war, irgendwelche Zahlen, Fakten oder Vorgänge zu erfinden. ${ }^{29}$

Beide Vorworte sind je für sich bemerkenswert: Das erste Vorwort würde in letzter Konsequenz bedeuten, dass durch das Eingeständnis, dass Teile erfunden sind, der Referenzcharakter des gesamten Textes in Frage gestellt wird, dass also auch die auf Dokumenten basierenden Fakten in ihrem Geltungsanspruch relativiert würden. Das zweite Vorwort reklamiert dann wiederum, dass es sich letztlich um einen faktualen Text handelt, trotz aller satirischen Verfremdungseffekte. In beiden Fällen wird die Hybridität der Dokumentarsatire negiert.

Auch das Gericht negierte die Hybridität des Textes und nahm im Verlauf des Verfahrens eine Trennung in satirische und dokumentarische Textpassagen vor. Diese Trennung des Textes kritisiert Delius als eine „Scheinlogik“ des Gerichtes, die zudem auch der Rechtsprechung des Bundesverfassungsgerichtes widerspreche, so der Autor in der ausführlichen Dokumentation des Gerichtsverfahrens, das der Neuauflage des Rotbuch Verlages von 1995 nachgestellt ist. ${ }^{30}$ In der Praxis des Verfahrens führte dies dazu, dass zunächst jeder Satz und jede Behauptung danach bewertet wurde,

ob sie Tatsachenbehauptungen oder Satiren sind. Wenn ironische oder satirische Wendungen und vor allem keine Zahlen vorliegen, gilt die Behauptung als satirisch, damit als Kunst. Wenn ,lediglich` Fakten oder präzise Angaben vermittelt werden, gilt das als Tatsachenbehauptung. ${ }^{31}$

Letztlich interpretierte das Gericht die satirischen Passagen damit als rein fiktionale Textabschnitte, die Tatsachenbehauptungen als faktuale. Von den von Delius grob geschätzten rund „4000 Zahlen, Fakten- und Datendetails“ des Buches wurden von den Siemens-Anwälten „19 angeblich falsche Sätze, Halbsätze, Wörter oder Prozentzahlen“ identifiziert; ${ }^{32}$ sprich, vor Gericht wurde am Ende lediglich die Faktenwahrheit dieser 19 Textstellen verhandelt.

An dieser Stelle sei ein etwas grundlegenderer erzähltheoretischer Exkurs angeführt: Generell unterscheidet die Erzähltheorie bekanntlich die beiden Ebenen des Geltungsanspruches bzw. des „pragmatischen Status“33 eines Textes, der entweder faktual oder fiktional und ausschließlich aus paratextuellen Informatio-

29 Delius: Unsere Siemens-Welt, 11.

30 Delius: Unsere Siemens-Welt, 175-252, hierzu insbesondere 202.

31 Delius: Unsere Siemens-Welt, 201-202.

32 Delius: Unsere Siemens-Welt, 206.

33 Martínez, Matías/Scheffel, Michael. Einführung in die Erzähltheorie. München: C.H.Beck 2019, 16. 
nen zu entnehmen ist, vom ontologischen Status des dargestellten Inhaltes, der entweder fiktiv oder real ist. Matías Martínez und Michael Scheffel verwenden in diesem Zusammenhang in ihrer Einführung in die Erzähltheorie für faktual bezeichnenderweise auch das Synonym „authentisch“. ${ }^{34}$ So wichtig und grundlegend diese Differenzierung ist, so wenig sagt sie doch über den Status eines Textzitates, eines Papierblattes, eines Filmausschnittes oder einer Audioaufnahme als Dokument aus. Wenn in einem faktualen Text ausschließlich von realen Fakten berichtet wird, heißt dies noch lange nicht, dass uns auch historische authentische Dokumente präsentiert werden. Unsere Siemens-Welt kommt etwa durchgehend ohne Fußnoten oder explizite Verweise auf Dokumente oder andere Quellen aus. Während der Nachweis durch Dokumente in historiographischen Texten zum Beispiel eine grundlegende Arbeits- und Beweismethode ist, müssen wir den Behauptungen von Texten in Autobiografien oder Dokumentationen in der Regel eher Glauben schenken (oder genauer: uns mit der Autorisierung durch die Autorinstanz begnügen), da in letzteren der Darstellungsmodus nicht der des Beweisens, sondern eher der des narrativen Nacherzählens oder Behauptens ist. W. G. Sebald zum Beispiel führt in seinem Werk diesen naiven Dokumentenglauben an der Nase herum, wenn die in seinen Texten eingebauten historischen fotografischen Dokumente gerade nicht das zeigen, was im Text behauptet wird, das sie belegen. ${ }^{35}$

Je nachdem, ob man das ,Doku' im Kompositum Dokufiktion als genetivus subjectivus oder objectivus meint, kann damit die Verwendung von Dokumenten in fiktionalisierenden Erzählformen oder das Darstellungsverfahren des Dokumentarischen als Unterbegriff des faktualen Erzählens gemeint sein. Da der Dokumentenbegriff hierbei wesentlich ein Aspekt der medienmateriellen Ebene zu sein scheint, kann man auch von der Dichotomie zwischen authentischem Dokument vs. vermittelndem Diskurs (oder kurz Dokument vs. Diskurs) sprechen. Um in diese Dichotomie sodann noch die ,Fiktion‘ zu integrieren, mag die Typologie von Christian Klein und Matías Martínez helfen, die in ihrem Sammelband Wirklichkeitserzählungen vier mögliche Grenzfälle zwischen Fiktivität und Fiktionalität unterscheiden: neben faktualen Erzählungen mit fiktionalisierenden Erzählverfahren und faktualen Erzählungen mit fiktiven Inhalten sind das noch fiktionale Erzählungen mit faktualen Inhalten und fiktionale Erzählungen mit faktualem Redemodus. ${ }^{36}$ Alle vier Formen können somit noch weiter danach unterschieden werden, ob in ihnen die Inhalte mit authentischem Medienmaterial - also mit Do-

34 Vgl. Martínez/Scheffel: Einführung in die Erzähltheorie, 16.

35 Vgl. hierzu auch den Beitrag von Bernd Stiegler in diesem Band.

36 Klein, Christian/Martínez, Matías. „Wirklichkeitserzählungen. Felder, Formen und Funktionen nicht-literarischen Erzählens“. In: Wirklichkeitserzählungen. Felder, Formen und Funktionen 
kumenten, Bildern, Filmen, Audioausschnitten, etc. - oder lediglich inhaltlich, d. h. diskursiv vermittelt präsentiert werden. Lediglich im zweiten Fall ist eine solche weitere Unterscheidung nicht ohne Weiteres notwendig.

Um damit auf ,unseren' Fall Delius zurückzukommen: Unsere Siemens-Welt ist ein Text, der über keine paratextuelle Gattungskennzeichnung verfügt, sich im Untertitel aber als Festschrift auszeichnet. Da Unsere Siemens-Welt der dokumentarischen Literatur der 1970er Jahre zuzurechnen ist, sind die Fiktionsmarker etwas schwächer ausgeprägt, als dies tendenziell in zeitgenössischen Dokufiktionen zu beobachten ist, aber im durchweg ironisierenden Stil des panegyrischen genus grande sind sie dennoch deutlich erkennbar. Im Schema von Klein/Martínez ist Unsere Siemens-Welt damit eine faktuale Erzählung mit fiktionalisierenden Erzählverfahren, die den faktual-authentischen Inhalt diskursiv vermittelt. Oder noch grundsätzlicher: Während auf „der Ebene der histoire [...] real verbürgte[ ] Ereignisse[], Konstellationen oder Personen" beschrieben werden, finden sich die „fiktionalen Darstellungsweisen“ vor allem auf der discours-Ebene ${ }^{37}$ - in dieser Hinsicht zeitgenössischen Dokufiktionen sehr ähnlich. Wobei diese Kennzeichnung eben nur bis zur Gerichtsverhandlung zutrifft. Ab der Auflage von 1976 ändert sich mit der gerichtlich angeordneten Schwärzung einiger kurzer Passagen zwar nicht der Geltungsanspruch und auch nicht der ontologische Status des Beschriebenen, sehr wohl aber der Dokumentenstatus des Textes.

Von den 19 ,vor Gericht stehenden“ Textstellen seien an dieser Stelle lediglich zwei näher betrachtet, die aber gleichsam exemplarisch die Frontstellung der beiden Parteien zeigen. Denn der Siemens-Delius-Prozess ist ein sehr zeitgebundenes Phänomen und nur vor dem Hintergrund des bundesrepublikanischen Klassenkampfes zwischen links-aktivistischer Dokumentarliteratur und der auf einer strengen Hierarchie basierenden kapitalistisch-patriarchalen Unternehmenskultur der 1970er zu verstehen. So scheint Siemens vor allem deshalb Klage gegen Delius' Buch eingereicht zu haben, weil man erstens Schaden für die „,Haus'-Ideologie“ bzw. die von oben gesteuerte interne Unternehmens- und Arbeiterkommunikation sowie zweitens generell einen Image-Verlust fürchtete. ${ }^{38}$

nicht-literarischen Erzählens. Hg. Christian Klein, Matías Martínez. Stuttgart/Weimar: J. B. Metzler 2009, 1-13, hier 4-5 [i. O. mit Herv.].

37 Diese Konstellation sei ein Kernmerkmal von Dokufiktion, so Bidmon: „Geschichte(n) zwischen Fakt und Fiktion“, 64.

38 So auch Delius in der Dokumentation des Verfahrens, wobei er noch als zwei weitere von insgesamt vier Gründen für die Klage anführt, dass sich führende Siemens-Manager sowie Teile der Siemens-Familie persönlich beleidigt fühlten und Delius schließlich auch darüber spekuliert, dass die Chefriege schlicht ein Exempel an ihm statuiert sehen wollte. Vgl. Delius: Unsere Siemens-Welt, 186-187. 
Unter den am Ende geschwärzten Stellen finden sich denn auch solche, die auf den ersten Blick denkbar banal erscheinen (vgl. Abb. 1, die Schwärzungen sind hier im Zitat durch graue Balken kenntlich gemacht):

\begin{abstract}
Andere Sparmaßnahmen seien hier als Beispiel dafür genannt, daß auch eine Weltfirma, die Milliarden-Umsätze macht, mit jedem Pfennig rechnen muß, auch wenn er von den eigenen Mitarbeitern kommt. Wenn wir beispielweise nach der Ankündigung von Bierpreiserhöhungen Zehntausende von Bierkästen aufkaufen und nachher zum neuen höheren Preis an die Mitarbeiter verkaufen und damit die Kassen des Hauses um 1 DM pro Kasten Bier stärken. Wenn wir Broschüren wie „Begegnung mit Siemens“, die wir an Besucher des Münchner Siemens-Museums kostenlos verteilen, unseren Mitarbeitern für 5,50 DM anbieten. ${ }^{39}$
\end{abstract}

Nicht nur vom Bierpreis, auch vom Preis für die Bockwurst hing damals also angeblich der interne Betriebsfrieden ab, beides diente der Unternehmensführung als Hebel für die Steigerung der Arbeitsmoral. Zumindest hat Siemens während des Verfahrens wiederholt versucht, das Gericht davon zu überzeugen, dass „der Bierpreis neben dem Preis für die Bockwurst als der eigentliche Indikator für die soziale Einstellung der Unternehmensleistung gilt und deshalb regelmäßig Gegenstand besonders intensiver Verhandlungen mit dem Betriebsrat ist (OLG 137)“.40 Dass eine solche Bierpreispolitik überhaupt das Potenzial der Zersetzung der Arbeitsmoral haben und deshalb den Betriebsfrieden gefährden könnte, ebenso wie der letztlich gänzlich kleinbürgerliche und unsouveräne Umgang damit, dass dies in einer Satire publik gemacht wurde - Siemens hat auch versucht, die Verbreitung von Gewerkschaftspublikationen, in denen Unsere Siemens-Welt rezensiert wurde, zu unterbinden -, wirkt heute wie eine realsatirische Antwort auf die reale Satire.

Die gerichtlich angeordneten Schwärzungen betreffen indes nicht nur solche vermeintlich banalen Informationen, sondern durchaus auch Textstellen deutlich gravierenderen Inhalts, wie z. B. die folgende:

Da nicht wenige von diesen in die Konzentrationslager eingewiesen wurden, herrschte eine für die kontinuierliche Produktion nicht sehr förderliche Fluktuation. So fügte es sich z. B., daß die 2000 Häftlinge und Fronarbeiter, die Anfang 1943 von Siemens zur Installierung des großen Vergasungskrematoriums im KZ Auschwitz eingesetzt waren, in eben diesem Krematorium ihr Ende fanden, und so verstummten auch die Zeugen für die Beteiligung des Hauses an diesen unerfreulichen Zeiterscheinungen. ${ }^{41}$

39 Delius: Unsere Siemens-Welt, 100.

40 Hier zitiert nach dem Anhang in: Delius: Unsere Siemens-Welt, 207.

41 Delius: Unsere Siemens-Welt, 48. 
Auch dass Siemens mit allen Mitteln versucht, seine Verstrickung an dem Menschheitsverbrechen der Shoa nicht an die Öffentlichkeit kommen zu lassen, ist Zeugnis einer Firmenpolitik, die typisch für die ,Erinnerungskultur dieser Phase der deutschen Nachkriegszeit ist. Inzwischen sind die großen deutschen Konzerne eher dazu übergegangen, die eigene Firmengeschichte von Historikerkommissionen aufarbeiten zu lassen. ${ }^{42}$ Nach Auffassung des Gerichtes haben die von Delius präsentierten Dokumente und Quellen „nicht ausgereicht“43, um diese Aussagen zu belegen und mussten daher geschwärzt werden. Wobei dies keineswegs bedeutet, dass diese Aussagen nicht zutreffend sind, das Gericht hielt in fragwürdiger Weise lediglich Verweise auf Publikationen, die in der DDR erschienen waren, für nicht glaubwürdig genug, um als Belege, sprich Dokumente zu gelten.

Auch in diesem Zusammenhang entpuppte sich das ganze Gerichtsverfahren als ein großes PR-Desaster für Siemens. Gegen Veröffentlichungen zu ihren Verstrickungen in die Nazi-Apparatur im Allgemeinen wie in den Bau von Konzentrationslagern im Besonderen war Siemens seit 1945 immer wieder gerichtlich vorgegangen, ${ }^{44}$ insofern war auch dieser Prozess nur eine konsequente Weiterführung der bisherigen PR-Strategie. Doch einerseits wurde auch und gerade im Zuge der Recherchen zum Prozess eine Fülle von Quellenmaterial zutage gefördert, die das ganze Ausmaß der ,intensive[n] Zusammenarbeit der Siemens-Firmen mit dem Nazi-Staat und der Siemens-Größen mit den Nazi-Größen“ bis hin zum Nachweis einer „faschistischen Ideologie im Hause“ sowie auch den Umfang der „Beteiligung an Vernichtungslagern“ deutlich machten. ${ }^{45}$ Und andererseits wird auch an vielen weiteren Stellen in Unsere Siemens-Welt vergleichbar Schreckliches und werden ähnliche schwerwiegende Verstrickungen in Kriegshandlungen oder gar Kriegsverbrechen geschildert. Generell ist es das Kernnarrativ der Satirefestschrift, nachzuzeichnen, dass der Aufstieg der Siemens-Familie wie des Konzerns überhaupt erst durch weitverzweigte Verwicklungen in Kriegsverbrechen und -geschäfte möglich wurde. Die Schilderungen dieser Kriegsverbrechen und Kriegsgewinne an all jenen Textstellen, die weiterhin lesbar gedruckt werden durften, waren damit durch das Gerichtsverfahren jedoch nunmehr ,höchstrichterlich' auf ihre Richtigkeit hin überprüft und damit gleichsam als historisch verbriefte Fakten autorisiert.

Dieser Aspekt ist für die erzähltheoretische Bewertung des ontologischen Status des Textes sehr bedeutsam: Erst das Urteil macht aus der diskursiv mit fak-

42 Wobei es auch heute freilich noch zahlreiche unrühmliche Versuche der Geschichtsklitterung gibt, besonders perfide etwa im Fall des Coburger Brose-Konzerns und der Stoschek-Familie.

43 Delius: Unsere Siemens-Welt, 207.

44 Vgl. Delius: Unsere Siemens-Welt, 210.

45 Delius: Unsere Siemens-Welt, 211. 
tualem Geltungsanspruch vorgetragenen Erzählerrede ein durch ein Gericht autorisiertes und authentifiziertes Dokument. Der für den Leser einzige erkennbare Ausdruck dieser gerichtlichen Autorisierung des Inhaltes sowie der Authentifizierung des Textes als Dokument - sozusagen gleichsam das gerichtliche Siegel sind aber eben diese Schwärzungen. Wir nehmen in der Rezeption damit automatisch einen ,richtigen Umkehrschluss` vor, indem wir alles, was nicht geschwärzt ist, als richterlich autorisierte und authentifizierte Fakten erkennen. Dass der Text Unsere Siemens-Welt durch das Gerichtsverfahren den Status als Dokument erhält, ändert also den Textstatus. Bei Dokufiktionen ist ja generell die spezifische Hybridität der Texte zwischen Fakt und Fiktion zu konstatieren. Im vorliegenden Fall kann aber näher unterschieden werden zwischen dem Geltungsanspruch des Textes, der bei Delius ja schon vor dem Gerichtsverfahren der eines faktualen Textes war, und dem eigentlichen Textstatus, der nach dem Prozess und durch die Schwärzung für den Rezipienten deutlich erkennbar der eines Dokumentes ist.

\section{Jan Brandts Tod des Autors in Turin}

Im zweiten Beispiel, das hier näher betrachtet werden soll, stellen sich die Textschwärzungen in jeglicher Hinsicht als ein anderes Phänomen dar. Jan Brandts Tod in Turin ist als Autofiktion ebenso ein Hybridtext zwischen Fakt und Fiktion, beide Rezeptionsverträge werden schon in den Paratexten geschlossen. Die Selbstkennzeichnung als „Materialband“ und „Turiner Journal“ etwa kennzeichnen den Text als autobiographisch und faktual, der Titel Tod in Turin wie der Untertitel Eine italienische Reise ohne Wiederkehr sind hingegen deutliche Fiktionsmarker - nicht nur inhaltlich, denn Jan Brandt ist ja lebend aus Turin zurückgekommen, sondern auch in ihrer Anspielung auf Thomas Mann und generell auf literarische Italienreisen, in deren Genealogie stehend sich der Text sehr ausführlich selbstinszeniert. ${ }^{46}$ Wie immer in autofiktionalen Texten haben wir es auch in diesem Fall mit einer zumindest namentlichen Übereinstimmung von Autor,

46 Diese Selbstinszenierung wird erkennbar ironisch schon im Peritext vorgeführt: So heißt es etwa auf der Umschlagrückseite, dass ,alle deutschsprachigen Schriftsteller von Weltrang [...] über ihre italienische Reise geschrieben“ haben. Sekundiert wird diese ironische Geste durch eine aufklappbare Umschlagsillustrierung, die Tischbeins berühmtes Gemälde Goethe in der römischen Campagna (1787) neben einer Comiczeichnung zeigt, die Brandt in derselben Pose portraitiert. Diese Selbstinszenierung und Ironisierung ist keine Kleinigkeit, wenn man Goethes Italienische Reise nicht nur als Fortsetzung von Goethes Lebensbericht, sondern, in einer Formulierung Wagner-Egelhaafs, als die Feier seiner „Wiedergeburt als Autor“ versteht. Textintern wird dies noch weitergeführt: Das zweite Kapitel besteht ausschließlich aus 20 Seiten lang angeführten Zi- 
Hauptfigur und Ich-Erzähler zu tun; allerdings nur vermeintlich, denn anders als in Autobiografien, in denen mit ein und demselben Namen die Authentizität des Beschriebenen autorisiert und beglaubigt wird, liegt in Autofiktionen in der Regel nur eine fingierte Namensidentität vor. Das Beschriebene wird dadurch gerade nicht beglaubigt, vielmehr müssen der empirische Autor Jan Brandt, der IchErzähler „Jan Brandt“ und die Hauptfigur „Jan Brandt“ als drei voneinander zu trennende Entitäten aufgefasst werden.

In Tod in Turin beschreibt Jan Brandt seine Reise zur Turiner Buchmesse, auf der die Übersetzung seines Debüterfolgs Gegen die Welt (2009) vorgestellt und beworben wurde, es geht also darum, was es bedeutet, als junger Erfolgsautor den ,Marktplatz Literatur ${ }^{`}$ zu betreten. Tod in Turin ist entsprechend eine Art Autorschafts-Bildungsroman; Brandt beschreibt darin gewissermaßen das coming-of-age seiner Autorschaft als eine Art ,Ankunftsroman', der Brandts Eintritt und ,Ankommen' im literarischen Betrieb thematisiert. Gerade diese Indienstnahme durch den Betrieb mit allen damit verbundenen Erwartungshaltungen und Projektionen des Publikums will sich der Jan Brandt' dieser Geschichte beständig entziehen. Anders formuliert: Der Ich-Erzähler versucht sich der Autorisierungsfunktion zu entziehen, die hinter dem Konzept einer emphatisch verstandenen oder rezipierten Autorschaft steckt. Was damit verweigert wird, ist letztlich natürlich auch die Vorstellung einer referenziellen oder relationalerschreibbaren Authentizität. Tod in Turin erzählt somit den fingierten und von der Hauptfigur herbeigesehnten Tod des Autors (oder der Autorfunktion) ,Jan Brandt' in Turin.

Diese Verweigerung der Autorisierungsfunktion einerseits und von Authentizitätsvorstellungen andererseits lässt sich unter anderem an dem Spiel mit Textschwärzungen erkennen, das in Tod in Turin vorgeführt wird. Im Lektorenvorwort - es ist mit „Martin Kordić, Lektor, Köln im Frühjahr 2015“ unterschrieben wird die Notwendigkeit von Schwärzungen wie folgt erläutert:

Aus persönlichkeitsrechtlichen Gründen sind einige Namen und einige biografische Angaben geschwärzt oder verändert worden. [...] Einige sagen, dass sie das, was sie hier sagen, so nie gesagt haben - so höflich, nett und auskunftsfreudig. Andere finden, Jan Brandt habe sie insgesamt viel zu positiv dargestellt, in Wirklichkeit seien sie ganz anders, härter, böser, dämonischer. Daher möchte ich empfehlen, diese Reisereportage als Erzählung anzusehen, eine Fiktion voller Fakten. ${ }^{47}$

taten von deutschsprachigen Autor^innen und ihren Italienreisen, gefolgt von einem „Und jetzt ich. "Vgl. Brandt: Tod in Turin, 70-89.

47 Brandt: Tod in Turin, 5. 
Nichts an diesem Vorwort ist erhellend, im Gegenteil, mit jedem weiteren Satzteil wird die Sache nur noch komplizierter oder - im Hinblick auf den hybriden Textstatus ist dies natürlich bedeutsam - zunehmend indifferent und unentscheidbar. Die Funktion des gesamten Vorwortes ist letztlich nur darin zu sehen, den Hybridstatus des Textes zwischen Fiktion und Faktendarstellung zu markieren, davon abgesehen ist der vorgebliche Inhalt, eine juristische Absicherung zu liefern, gänzlich unnötig, weshalb es als Lektorenvorwort ein bloß fingiertes ist. Nur wird es als solches freilich nicht wahrgenommen, nicht nur die räumlich-zeitliche Verortung und die rhetorische Konvention der Unterschrift erzeugen einen Authentizitätseffekt, ein solches Vorwort ist nur bei faktualen Texten überhaupt nötig. D. h. was hier ebenfalls erzeugt wird, ist der Rezeptionseffekt, dass dem Rezipienten alles nachfolgend Beschriebene als das Ergebnis einer gründlichen Recherche und Dokumentation erscheint.

Aber damit immer noch nicht genug: Was ebenso lediglich fingiert ist, sind die persönlichkeitsrechtlichen Gründe, die hier als Begründung für die Schwärzung angeführt werden. Denn erstens hätte Brandt in einer Autofiktion ja auch die Wahl gehabt, die Namen einfach zu ändern oder das Dargestellte anders zu erzählen. Die Schwärzungen sind also natürlich vollkommen unnötig, aber dadurch freilich ein umso bewusster gewähltes Stilmittel. Zweitens wird die Schwärzung auch deshalb als ironisches Spiel mit der persönlichkeitsrechtlichen Klagefreude - die letztlich immer eine Beschränkung der Freiheit der Kunst darstellt - vorgeführt, weil selbstredend noch niemand in der Weltgeschichte des Persönlichkeitsrechtes aufgrund einer zu positiven, zu höflichen und zu netten Darstellung seiner Person geklagt hat. Man könnte es fast als eine Ironisierung der Cancel Culture interpretieren - avant la lettre, so müsste man freilich hinzufügen, denn so alt ist der Text inzwischen auch schon wieder bzw. so schnell entwickeln sich derzeit neue Diskursregeln in der Gegenwartskultur. Und drittens wird hier in aller Deutlichkeit auch noch gänzlich verunklart, auf wen und auf welche Instanz die Schwärzung in Tod in Turin überhaupt zurückgeht: Erfolgt sie aufgrund einer bloß antizipierten Klageandrohung, sprich in vorauseilendem Gehorsam, oder gab es eine solche Androhung bereits, schließlich scheint Kordić hier ja Reaktionen der im Text beschriebenen Personen zu referieren. Vor allem aber ist vollkommen unklar, ob die Schwärzungen auf richterliche Anordnung zurückgehen - die Begründung „[a]us persönlichkeitsrechtlichen Gründen“ ruft zumindest juristische Argumentationsrhetorik auf -, oder ob Kordić mit seiner Unterschrift und in Köln im Frühjahr 2015 im Zuge seines Lektorats die Schwärzungen vorgenommen hat. Und selbst wenn wir Jan Brandt als Urheber des Anonymisierungsspiels annehmen, wissen wir immer noch nicht, ob damit der empirische Autor oder die Erzählinstanz dafür verantwortlich zeichnet. Im Text selbst äußert sich die Schwärzung dann wie folgt (vgl. Abb. 2): 
Wie sich herausgestellt, hat an der studiert. Sie habe, sagt sie,

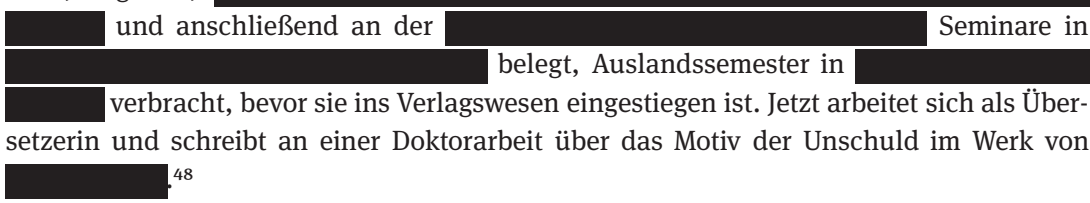

Deutlich erkennbar handelt es sich hier vor allem um ein ironisches Spiel mit der Schwärzungstechnik, zu leicht hätte diese Passage auch schlicht anders umschrieben werden können. Anders formuliert, es geht Brandt hier vor allem darum, diese Technik als solche in seinem Text auszustellen. Der Rezeptionseffekt, der damit erzeugt wird, ist, dass wir - mit Huck gesprochen - zunächst einen falschen Umkehrschluss vornehmen. Denn wir nehmen an, dass hier alles ohne autorschaftliche Filterung präsentiert wird, sogar in einer derartigen protokollarischen Unmittelbarkeit, dass das, was nicht für die Öffentlichkeit bestimmt ist, geschwärzt werden muss. Eigentlich muss in einer Autobiografie die Autorinstanz das Erzählte verbürgen, muss es autorisieren. Aber diese Autorautorisierung oder Autorauthentifizierung verweigert Brandt hier gerade, und das ostentativ. Indem die Schwärzung als von einer unabhängigen Instanz vorgenommen fingiert wird (siehe Lektorenvorwort), erzeugen wir in der Rezeption den falschen Umkehrschluss, dass wir den Text als ein authentisches Dokument wahrnehmen, obwohl er das nicht ist.

Noch einmal etwas anders und stärker auf die Ausgangsfragestellung hin formuliert: Brandt führt mit der Schwärzungstechnik ein ironisches Spiel mit der Materialität des Textes und damit mit dem Textstatus als Dokument vor. Wenn uns geschwärztes Textmaterial präsentiert wird, so der unmittelbar einsetzende Rezeptionseffekt des falschen Umkehrschlusses, dann haben wir es mit der ungefilterten Präsentation eines Dokumentes zu tun. Die Erzähl- und Autorinstanz nimmt sich in dieser Fingierung der ungefilterten Präsentation maximal zurück, ja man könnte in letzter Konsequenz sogar davon sprechen, dass der Erzähler sich hier wie ein bloßer Herausgeber geriert. Erneut ein paradoxer Hybriditätseffekt, denn während die autobiographischen (und auch noch die deutlich autofiktionalen) Passagen eine große Nähe zur Erzählinstanz und Hauptfigur erzeugen, der wir zudem beständig in interner Fokalisierung und Autodiegese folgen, erzeugt die Schwärzung durch die Fingierung einer Herausgeberfiktion eine maximale Distanz zu allem, was eigentlich in der Ich-Form beschrieben wird. An dieser Stelle sei nur kurz erwähnt, dass sich diese Aspekte auch noch in anderen Gestaltungs-

48 Brandt: Tod in Turin, 199. 
merkmalen des Textes zeigen: Die beständige Selbstironisierung der eigenen Autorautorität ist ein durchgängiges, wenn nicht das zentrale Thema in Tod in Turin, es lässt sich etwa auch an Autorschaftsallegorien und Reflexionen über das Erzähler-Ich erkennen. ${ }^{49}$

Eine der Schwärzung vergleichbare Technik findet sich in Tod in Turin darüber hinaus in den über 180 Fußnoten, die fortlaufend eingebaut sind. Dieses Spiel mit Fußnotenverweisen ist nicht nur deshalb bedeutsam, weil es sich auf eine lange literarische Tradition bezieht und entsprechend von besonderer literaturhistorischer Dignität ist - man denke etwa Jean Pauls Leben des Quintus Fixlein (1796). Wobei die Fußnoten bei Jean Paul allerdings eine Komplexitätssteigerung der Erzählebenen und -instanzen bewirken. Der Effekt der Fußnoten bei Jan Brandt ist dagegen konträr zur Verwendung in wissenschaftlichen Arbeiten, wo sie der Autorisierung von Faktenwissen dienen. In ihnen wird, von wenigen Ausnahmen abgesehen, ausschließlich textintern schon Bekanntes oder bloßes Wikipedia-Wissen paraphrasiert. Auch hier scheint dieses Verfahren also auf den ersten Blick den klassischen Autorisierungseffekt zu erfüllen und einer Beweisführung ex auctoritate zu dienen. Für diesen Autorisierungseffekt müsste es sich indes um angesehenere und autoritäre Quellen oder um authentische Dokumente oder Quellen handeln, und mindestens müsste durch die Fußnotentexte ein Erkenntnisgewinn generierbar sein. Doch durch die Banalität der Informationen erzeugen die Fußnoten ebenso wie die Schwärzungen einen konträren Effekt. In beiden Fällen, den Schwärzungen wie den Fußnoten, lässt sich also ein ironisches Spiel mit der Materialität des Textes erkennen, in beiden Fällen wird die Materialität von Dokumenten bloß fingiert. Auf den ersten Blick erzeugen beiden Verfahren einen referenziellen Authentizitätseffekt, auf den zweiten Blick wird hingegen deutlich, dass die Erzählinstanz dadurch eine sehr grundlegende Autorisierungsfunktion der Autorinstanz verweigert. Indem durch diese Verweigerungsgeste die Unmöglichkeit einer referenziellen oder relational erschreibbaren Authentizität inszeniert wird, entsteht wiederum ein metaauthentischer Rezeptionseffekt.

49 Vgl. Weixler, Antonius. „,Wenn ich doch nur vergessen könnte, wer ich bin. 'Antiautoritäre Autorschaft in Jan Brandts ,Tod in Turin“." In: Sich selbst erzählen. Autobiographie - Autofiktion Autorschaft. Hg. Sonja Arnold u. a. Kiel: Ludwig 2018, 379-397. 


\section{Florence Meunier oder Zur Erkennbarkeit verfremdet}

In Florence Meuniers The Man Who Agreed hat die Textschwärzung wieder eine andere Funktion als in den ersten beiden Beispielen. Wie eingangs bereits erwähnt, kürzt Meunier die Allgemeinen Geschäftsbedingungen von Apples Speicherdienst iCloud so zusammen, dass aus dem juristischen faktualen Text folgende fiktionale Geschichte wird:

\footnotetext{
This is the story of a man, / who one day was too busy / or maybe too lazy that he, too quickly, / clicked on I agree.

What the latter did not foresee

is that he could never again disagree.

The lesson of this story is / that one shall not concede, to something one does not read. ${ }^{50}$
}

Erneut haben wir es also mit einer Satire mit ausgeprägter Ironie zu tun. Formal ruft der Text sehr frei - durch den vom Original abweichenden Zeilenumbruch hier oben soll dies verdeutlicht werden - den Stil eines Limerick mit seiner fünfversigen Struktur mit scherzhafter, hier aber auch moralisch-didaktischer Pointe am Ende auf. Geschichte und Moral hängen hierbei unmittelbar mit den Geschäftsbedingungen zusammen, auf denen dieser Text basiert und aus dessen Material er besteht. Bedeutsam daran ist für unseren Zusammenhang selbstredend, dass die Schwärzung hier nicht etwas verbirgt, sondern vielmehr überhaupt erst etwas hervorbringt. Auf der ersten Ebene ist dies natürlich diese Geschichte, auf der zweiten Ebene werden darüber hinaus aber überhaupt erst die Tragweite und die juristischen Folgen - konkret die Unumkehrbarkeit der Einverständniserklärung, die später nicht mehr zurückgenommen werden kann - der Zustimmung zu diesem Dokument deutlich und erkennbar gemacht.

Oder, noch einmal anders und im Hinblick auf die zentrale Fragestellung nach dem Text- und Dokumentenstatus betrachtet: Meuniers Ausgangsmaterial ist ein Dokument, die Schwärzung führt hier nicht dazu, diesen Dokumentenstatus überhaupt erst zu erzeugen oder diesen in irgendeiner Hinsicht zu autorisieren oder authentifizieren, sondern, ganz im Gegenteil, die Schwärzung führt hier zunächst dazu, dem Text diesen Dokumentenstatus zu entziehen und zum bloßen Materiallieferanten zu degradieren. Im Ergebnis steht sodann ein satirisch-ironischer Text abstrakt-didaktischen Inhaltes, der damit auch jeglicher konkreten

50 Meunier: The Man Who Agreed. 


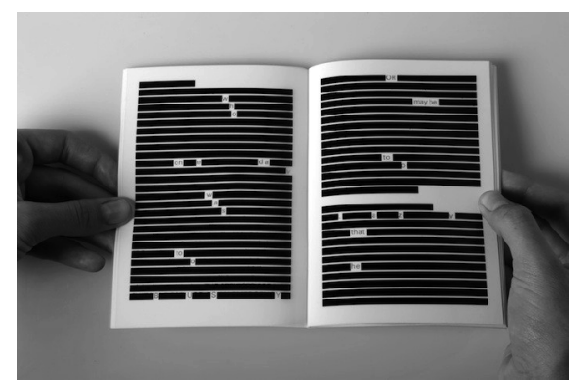

Abb. 5: Die Schwärzungen bringen in diesem Fall die Geschichte überhaupt erst hervor.

Fakten entbehrt. Es ist unmittelbar erkennbar, dass die limerickeske Kurzgeschichte kein Dokument ist, sondern ein literarischer Text. Paradoxerweise wird aber erst durch diesen Entzug des Dokumentenstatus deutlich und für jedermann verständlich und erkennbar, was es mit dem Dokument eigentlich auf sich hat. Das Dokument wird durch den schwärzenden Entzug des Dokumentenstatus zur Erkennbarkeit verfremdet.

\section{Kurzer Ausblick anstatt eines langen Fazits oder: Der double bind staatlich-institutioneller Schwärzung}

In diesem Beitrag wurde versucht zu untersuchen, welchen Einfluss ein und dasselbe Verfahren im Umgang mit der Materialität von Texten auf den Status von Dokumenten und Texten haben kann. Abschließend sei noch kurz auf die Folgen und den damit verbundenen double bind der quantitativ sicherlich verbreitetsten Art von Textanonymisierung durch Schwärzung eingegangen: Wann immer staatliche Institutionen dazu gezwungen werden, ihre Dokumente zu veröffentlichen, neigen sie dazu, alles, was ihrer Meinung nach ein Staatsgeheimnis ist, unkenntlich zu machen. Der Muller-Bericht oder Slahis Guantánamo Diary wurden als Beispiele, bei denen diese Technik besonders ausgeprägt angewandt worden war, eingangs schon genannt. Allerdings zeigen diese Beispiele, dass solche Schwärzungen auch noch einen weiteren Effekt haben können und dass der falsche Umkehrschluss und überhaupt das Zuschreibungsphänomen Authentizität auch problematisch sein können. Problematisch ist dies deshalb, weil sich spä- 
testens hier deutlich zeigt, was so falsch ist am „falschen Umkehrschluss“. ${ }^{51}$ Der erste damit zusammenhängende Fehlschluss ist es etwa anzunehmen, dass die massenmediale Berichterstattung per se verfälschend oder manipulierend ist, zumal es ja, wie gezeigt wurde, unmöglich ist, direkt und unmittelbar, d. h. referenziell-authentisch zu kommunizieren. Es ist, zweiter Fehlschluss, genauso falsch und sogar noch viel problematischer, anzunehmen, dass Erzählungen, die von den Massenmedien abweichen, nicht verfälschend sind oder sogar auf irgendeine Art und Weise authentischer sein könnten. Oft wird dies aber angenommen bzw. oft inszenieren sich Erzählungen aber genau so. Damit wird Authentizität aber zu einem (auch) problematischen und für Manipulationen anfälligen Phänomen. Genauer lässt sich diesbezüglich eine Strukturanalogie zwischen Narrativen, die Authentizität erzeugen, und Narrativen von Verschwörungserzählungen erkennen. Denn Verschwörungserzählungen inszenieren stets eine Grenzüberschreitung von einer massenmedialen Oberfläche, die alle sehen und die als manipulativ vorgeführt wird, hin zu einer hinter dieser Oberfläche vermeintlich zu entdeckenden Verschwörung, die nur für wenige Eingeweihte erkennbar ist. ${ }^{52}$ Darüber hinaus brauchen Verschwörungserzählungen in der Regel einen Fehler oder eine kausale Lücke in der offiziellen Erklärung, in die sie mit ihrer alternativen Erzählung hineinstoßen können. ${ }^{53}$ Schwärzungen werden in diesem Zusammenhang für die Verschwörungsgläubigen zum Signum von Manipulationen, die von staatlichen Institutionen oder von ,den Massenmedien“ vorgenommen werden, und diese Schwärzungen werden als Leerstellen dann wiederum zum Einfallstor für Verschwörungserzählungen.

51 Huck weiter zum falschen Umkehrschluss: „Wenn A, dann B. Ergo: Wenn nicht A, dann nicht B. Dass das aber natürlich so nicht stimmt, lässt sich an einem sehr einfachen Beispiel zeigen: Wenn ich Deutscher bin, dann bin ich Europäer. Ergo: Wenn ich kein Deutscher bin, dann bin ich kein Europäer. Während die affirmative Implikation im Beispiel zutrifft, ist die Negation offensichtlich unsinnig und lässt sich so nicht ableiten; im Sinne einer semantischen Logik, handelt es sich hier um eine ,einseitige Implikation'. Indem der Dokumentarfilm die weit verbreiteten Ressentiments gegenüber den Massenmedien aufruft und negiert, wird durch die Suggestion einer ,wechselseitigen Implikation` von der Tatsache abgelenkt, dass nicht allein die Verfahren der Massenmedien in der Lage sind, zu verfälschen.“ Huck: „Authentizität im Dokumentarfilm“, 251. 52 Vgl. Weixler, Antonius. „Make Control Great Again. Die narrative Konstruktion, eingeweihten Wissens in Verschwörungserzählungen“. In: Postfaktisches Erzählen? Post-Truth - Fake News Narration. Hg. Antonius Weixler u. a. Berlin/Boston: De Gruyter 2021, 119-148.

53 Vgl. Butter, Michael. „Nichts ist, wie es scheint“. Über Verschwörungstheorien. Berlin: Suhrkamp 2018, 77-83. 


\section{Literatur}

Assmann, Aleida. „Vier Grundtypen von Zeugenschaft“. In: Zeugenschaft des Holocaust. Zwischen Trauma, Tradierung und Ermittlung. Hg. Fritz Bauer Institut. Frankfurt a. M.: Campus 2007, 33-51.

Baudrillard, Jean. „Der Geist des Terrorismus. Herausforderung des Systems durch die symbolische Gabe des Todes“. In: Der Geist des Terrorismus. Hg. Peter Engelmann. Wien: Passagen Verlag 2002, 11-35.

Bidmon, Agnes. „Geschichte(n) zwischen Fakt und Fiktion. Formen und Funktionen dokufiktionalen Erzählens in Jugendbüchern von Dirk Reinhardt: Edelweißpiraten und Train Kids.“ In: Fakt, Fake und Fiktion. Jahrbuch der Gesellschaft für Kinder- und Jugendliteraturforschung 2019. Hg. Gabriele von Glasenapp u. a. http://www.gkjf.de/jahrbuch-2019-open-access/, 63-77.

Brandt, Jan. Tod in Turin. Eine italienische Reise ohne Wiederkehr. Köln: DuMont 2015.

Butter, Michael. „Nichts ist, wie es scheint“. Über Verschwörungstheorien. Berlin: Suhrkamp 2018.

Delius. F. C. Unsere-Siemens-Welt. Eine Festschrift zum 125jährigen Bestehen des Hauses S. Erweiterte Neuausgabe mit einem Anhang über den Prozeß, über die Kunst der Satire, die Menschenwürde des Konzerns, Bierpreise und den verlorenen Kredit des Hauses S. Mit einem Nachwort von Friedrich Christian Delius. Hamburg: Rotbuch 1995.

Funk, Wolfgang/Krämer, Lucia. „Fiktionen von Wirklichkeit - Authentizität zwischen Materialität und Konstruktion“. In: Fiktionen von Wirklichkeit - Authentizität zwischen Materialität und Konstruktion. Hg. Wolfgang Funk, Lucia Krämer. Bielefeld: transcript 2011, 7-24.

Haselstein, Ulla/Gross, Andrew S./Snyder-Körber, MaryAnn. „Introduction: Returns of the Real“. In: The Pathos of Authenticity. American Passions of the Real, Hg. Ulla Haselstein, Andrew S. Gross, MaryAnn Snyder-Körber. Heidelberg: Winter 2010, 9-32.

Huck, Christian. „Authentizität im Dokumentarfilm. Das Prinzip des falschen Umkehrschlusses als Erzählstrategie zur Beglaubigung massenmedialen Wissens“. In: Authentisches Erzählen. Produktion, Narration, Rezeption. Hg. Antonius Weixler. Berlin/Boston: De Gruyter 2012, 239-264.

Klein, Christian/Martínez, Matías. „Wirklichkeitserzählungen. Felder, Formen und Funktionen nicht-literarischen Erzählens“. In: Wirklichkeitserzählungen. Felder, Formen und Funktionen nicht-literarischen Erzählens. Hg. Christian Klein, Matías Martínez. Stuttgart/Weimar: J.B. Metzler 2009, 1-13.

Knaller, Susanne. „Genealogie des ästhetischen Authentizitätsbegriffs“. In: Authentizität. Diskussion eines ästhetischen Begriffs. Hg. Susanne Knaller, Harro Müller. München/Paderborn: Fink 2006, 17-35.

Knaller, Susanne. Ein Wort aus der Fremde. Geschichte und Theorie des Begriffs Authentizität. Heidelberg: Winter 2007.

Martínez, Matías. „Zur Einführung. Authentizität und Medialität in künstlerischen Darstellungen des Holocaust“. In: Der Holocaust und die Künste. Medialität und Authentizität von Holocaust-Darstellungen in Literatur, Film, Video, Malerei, Denkmälern, Comic und Musik. Hg. Matías Martínez. Bielefeld: Aisthesis 2004, 7-21.

Martínez, Matías/Scheffel, Michael. Einführung in die Erzähltheorie. München: C.H.Beck 2019. 
Meister, Andre. „Wir veröffentlichen den Abschlussbericht - ohne die Schwärzungen“. Netzpolitik.org vom 24.06.2017. https://netzpolitik.org/2017/geheimdienstuntersuchungsausschuss-wir-veroeffentlichen-den-abschlussbericht-ohne-dieschwaerzungen/ (01.03.2021).

Meunier, Florence. The Man Who Agreed. Ohne Verlagsangabe 2015. Zitiert nach: https: //blog.supertext.ch/2015/01/lesenswert-wie-aus-den-16-seitigen-icloud-agb-einekurzgeschichte-wurde/ (01.03.2021).

o. V. „Geschwärztes PDF-Dokument blamiert Pentagon“. In: DerStandard.de vom 02.06.2005. https://www.derstandard.de/story/2034120/geschwaerztes-pdf-dokument-blamiertpentagon (01.03.2021).

Röttgers, Kurt/Fabian, Reinhard. „Authentisch“. In: Historisches Wörterbuch der Philosophie. Hg. Joachim Ritter. Darmstadt: Wissenschaftliche Buchgesellschaft 1971, Bd. I, Sp. 691-692.

Slahi, Mohamedou Ould. Das Guantanamo-Tagebuch [Guantánamo Diary]. Stuttgart: Cotta 2015.

Weixler, Antonius. „Authentisches erzählen - authentisches Erzählen. Über Authentizität als Zuschreibungsphänomen und Pakt“. In: Authentisches Erzählen. Produktion, Narration, Rezeption. Hg. Antonius Weixler. Berlin/Boston: De Gruyter 2012, 1-32.

Weixler, Antonius. „,Wenn ich doch nur vergessen könnte, wer ich bin. 'Antiautoritäre Autorschaft in Jan Brandts ,Tod in Turin““. In: Sich selbst erzählen. Autobiographie - Autofiktion - Autorschaft. Hg. Sonja Arnold u. a. Kiel: Ludwig 2018, 379-397.

Weixler, Antonius. „Make Control Great Again. Die narrative Konstruktion ,eingeweihten Wissens ' in Verschwörungserzählungen“. In: Postfaktisches Erzählen? Post-Truth - Fake News - Narration. Hg. Antonius Weixler u. a. Berlin/Boston: De Gruyter 2021, 119-148.

Wiegandt, Markus. Chronisten der Zwischenwelten. Dokufiktion als Genre. Operationalisierung eines medienwissenschaftlichen Begriffs für die Literaturwissenschaft. Heidelberg: Winter 2017.

Zipfel, Frank. „Autofiktion. Zwischen den Grenzen von Faktualität, Fiktionalität und Literarität?“ In: Grenzen der Literatur. Zum Begriff und Phänomen des Literarischen. Hg. Simone Winko, Fotis Jannidis, Gerhard Lauer. Berlin/New York: De Gruyter 2009, 287-314. 